\title{
Robust Evolutionary Algorithm Design for Socio-Economic Simulation: A Correction
}

\author{
Floortje Alkemade • Han La Poutré • \\ Hans Amman
}

Accepted: 26 May 2008 / Published online: 13 July 2008

(C) The Author(s) 2008

Recently we have discovered an error in the implementation of the mutation operator in our earlier work on robust evolutionary algorithm design for socio-economic simulation (Alkemade et al. 2006, 2007). ${ }^{1}$ The original paper compared two commonly used approaches to socio-economic simulation. In the first approach parameter settings for the evolutionary algorithm are directly derived from the underlying economic model while in the second approach to social learning parameter settings are chosen so as to optimise evolutionary algorithm performance. Main conclusions of the original paper are that the first approach may hinder the performance of the evolutionary algorithm and thereby hinder agent learning, that is, that social learning evolutionary algorithms are able to overcome the so-called spite-effect and obtain high profit outcomes. These main conclusions are still confirmed when the error in the mutation operator is corrected. However, the convergence behaviour of some of the individual runs differs significantly from the (incorrect) results presented in the earlier papers. More specifically, in the corrected experiments we do not observe the same type of premature convergence in approach I. In this paper we present the corrected results. The average convergence behaviour for the two approaches is shown in Fig. 1, where we see convergence to the higher profit Cournot Nash outcome (at output 40) using approach II whereas approach I leads to the lower-profit competitive outcome (at output 50) for these set of EA parameters. The corrected results for the individual runs are shown in Fig. $2 .^{2}$ While these figures

\footnotetext{
1 We thank Ludo Waltman and Nees Jan van Eck for pointing out the error to us.

2 Corresponding to Fig. 4 in the original paper.
} 

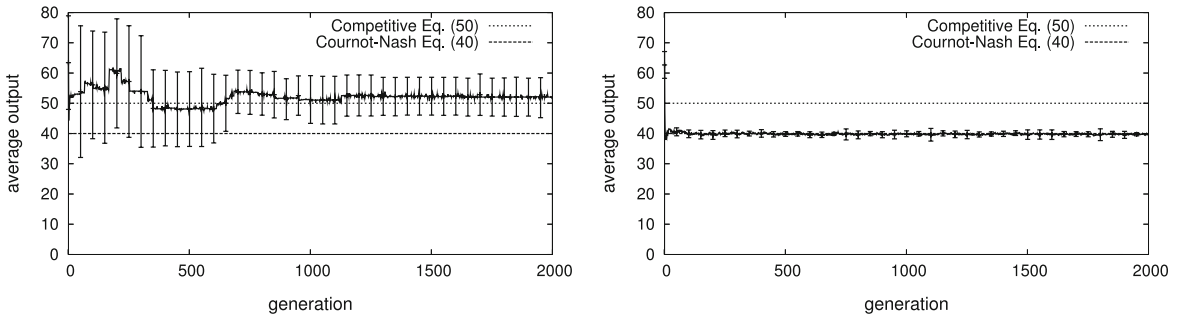

Fig. 1 Approach 1 (left) and approach 2 (right) with an initial density of 0.5 and a chromosomelength of 7
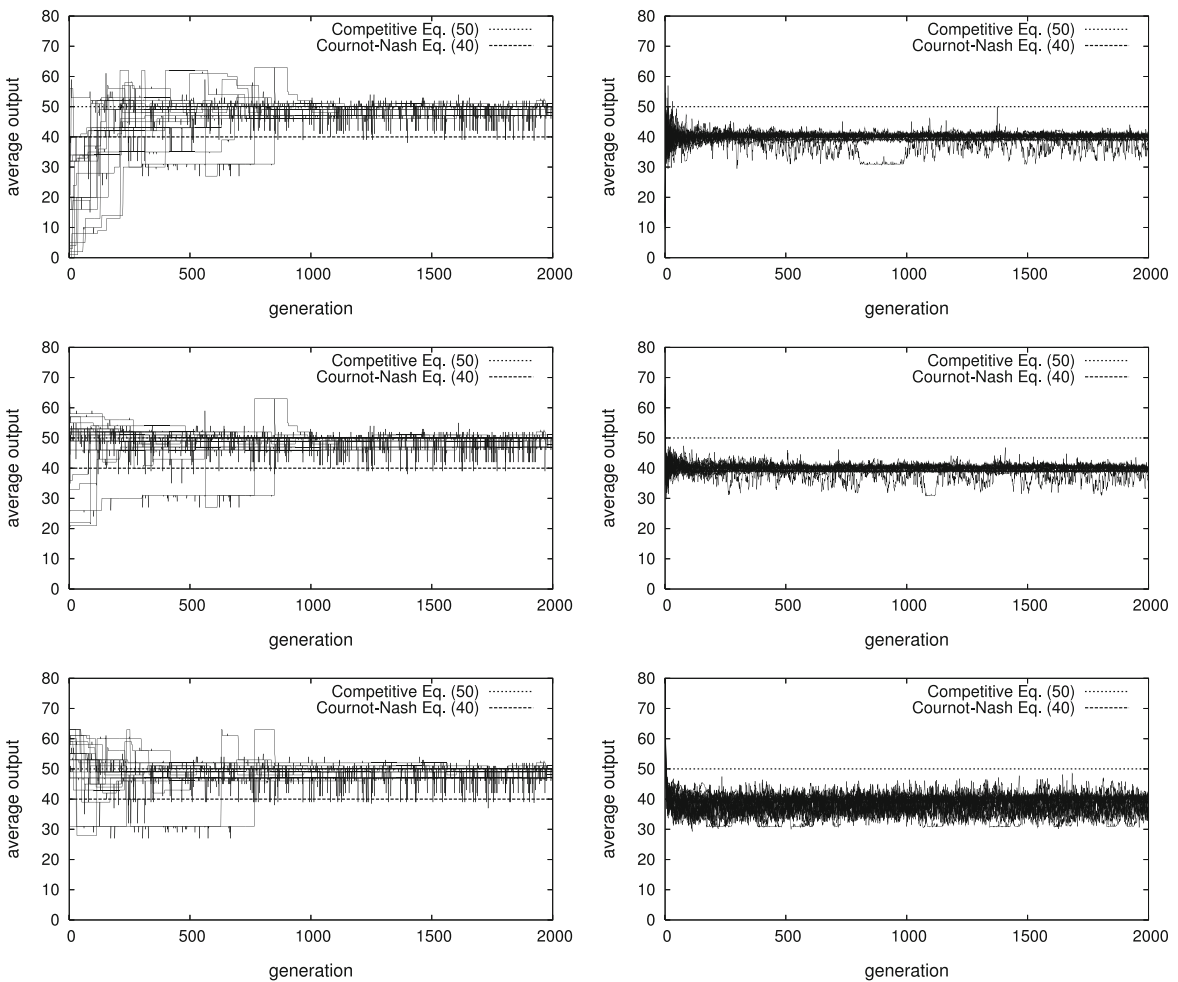

Fig. 2 Approach I (left) and approach II (right): individual runs for chromosomelength 6 (density $0.1,0.5$ and 0.9)

illustrate the robustness of approach II we do not observe the type of premature convergence that was depicted in our earlier (incorrect) results. As in the original paper we do see that, using approach I, we observe instability/premature convergence; the convergence behaviour differs for different values of the EA parameters such as initial density and chromosome length (see the corrected results in Fig. 3 for an illustration). 

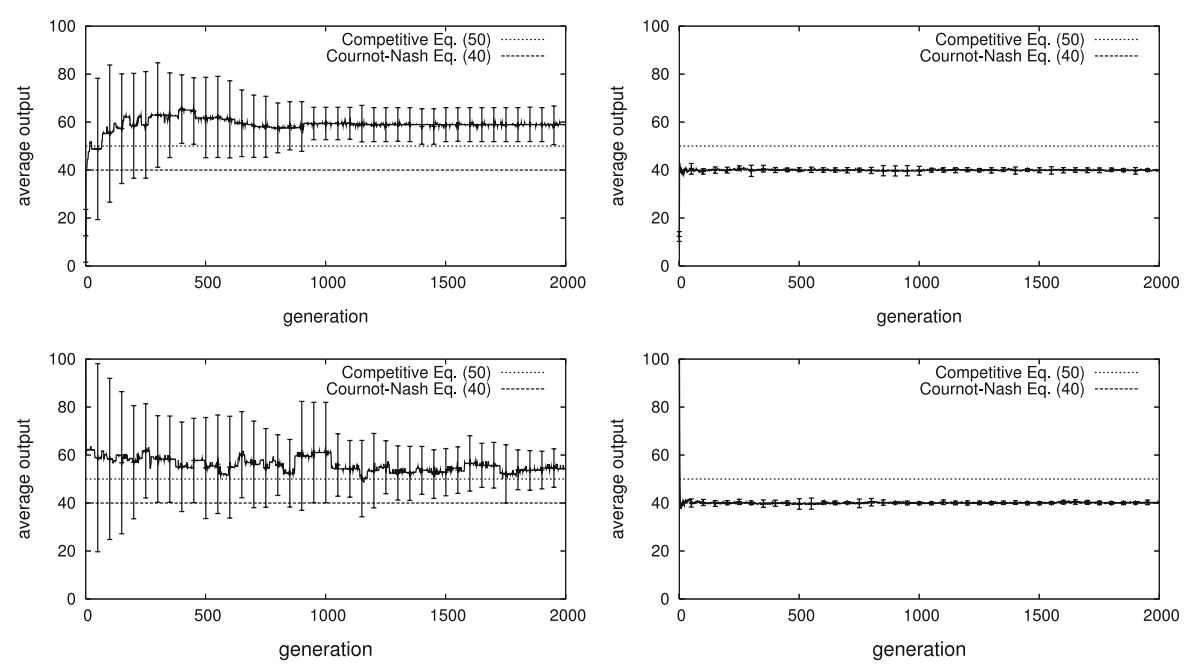

Fig. 3 Approach I (left) and approach II (right) with an initial density of 0.1 and a chromosomelength of 7 (top) and an initial density of 0.5 and a chromosomelength of 8 (bottom)

Open Access This article is distributed under the terms of the Creative Commons Attribution Noncommercial License which permits any noncommercial use, distribution, and reproduction in any medium, provided the original author(s) and source are credited.

\section{References}

Alkemade, F., La Poutré, J. A., \& Amman, H. M. (2006). Robust evolutionary algorithm design for socioeconomic simulation. Computational Economics, 28(4), 355-370.

Alkemade, F., La Poutré, J. A., \& Amman, H. M. (2007). On social learning and robust evolutionary algorithm design in the Cournot Oligopoly game. Computational Intelligence, 23(2), 162-175. 